\title{
Fabrication of Holder-type Piezoresistive Cantilever for Embryo Mass Measurement
}

\author{
Hayato Sone, ${ }^{1 *}$ Tomoyuki Kawakami, ${ }^{2}$ Akiko Saito, ${ }^{3}$ \\ Yuki Sayama, ${ }^{1}$ Sumio Hosaka, ${ }^{1}$ and Toshiya Sakata $^{3}$ \\ ${ }^{1}$ Graduate School of Science and Technology, Gunma University, 1-5-1 Tenjin-Cho, Kiryu, Gunma 376-8515, Japan \\ ${ }^{2}$ Graduate School of Engineering, Gunma University, 1-5-1 Tenjin-Cho, Kiryu, Gunma 376-8515, Japan \\ ${ }^{3}$ Department of Materials Engineering, Graduate School of Engineering, The University of Tokyo, \\ 7-3-1 Hongo, Bunkyo-ku, Tokyo 113-8656, Japan
}

(Received April 3, 2018; accepted October 3, 2018)

Keywords: cantilever-based biosensor, embryo mass, focused ion beam, assisted reproductive technology

A novel embryo evaluation system and microsensor devices have been developed using microcantilever-based biosensors. A holder-type piezoresistive cantilever was fabricated using a focused ion beam (FIB) to place and fix an embryo on the cantilever, and the monitoring system of the cantilever resonance frequency for measuring the embryo mass by the frequency sweep method was developed. The mass of a single 2-cell-stage mouse embryo was measured using the holder-type cantilever in a culture medium with calf serum. The resonance frequency of the cantilever before and after embryo placement was changed from 95.0 to $91.5 \mathrm{kHz}$. The resonance frequency was approximately constant during $20 \mathrm{~min}$ of monitoring. From the frequency change of $-3.5 \mathrm{kHz}$, the mouse embryo mass was estimated to be about $4.4 \mathrm{ng}$. Although this value is smaller than the mass calculated from the volume of the mouse embryo and the density of culture medium, it indicates the possibility of measuring a single mouse embryo mass using a holder-type cantilever sensor system.

\section{Introduction}

The development of a highly sensitive and compact biosensor for the evaluation of the activity of biomaterials, e.g., cells, fertilized eggs, and embryos, is demanded. In particular, the development of a quantitative evaluation technique is expected for assisted reproductive technology (ART) of in vitro fertilization (IVF) to select an embryo of good quality. Embryo selection in current human IVF is accomplished by qualitative morphological observation using an optical microscope because it is a noninvasive and effective method in predicting pregnancy. $^{(1,2)}$ Two kinds of quantitative selection methods for live embryos have been developed by two groups. The change in the elasticity of mouse zona pellucida is quantitatively evaluated during oocyte maturation, fertilization, and early embryo development stages using a micro-tactile sensor. ${ }^{(3,4)}$ The activity of a single mouse embryo is monitored in real time using a semiconductor-based field effect transistor sensor. ${ }^{(5,6)}$ Several researchers also

*Corresponding author: e-mail: hayatosone@gunma-u.ac.jp

https://doi.org/10.18494/SAM.2018.1951 
measured the mass of partial components in the embryo by mass spectrometry (MS), because the mass of an embryo is physiologically important in evaluating the living quality. Three bisbenzylisoquinoline alkaloids (liensinine, isoliensinine, and neferine) in the embryo were analyzed by liquid chromatography electrospray ionization tandem mass spectrometry (LC-ESIMS/MS) with a positive ionization mode. ${ }^{(7)}$ Lipid profiles of single oocytes and embryos were evaluated by mass measurement of phospholipid profiles using matrix-assisted laser desorption/ ionization mass spectrometry (MALDI-MS) fingerprinting. ${ }^{(8,9)}$ The characteristics of a single bovine embryo after cryopreservation were also measured by MALDI-MS. ${ }^{(10)}$ A single oocyte and single embryo lipid were also analyzed by a more direct and simpler MS technique of desorption electrospray ionization mass spectrometry (DESI-MS). ${ }^{(1)}$ However, as these mass measurements are based on destructive analysis techniques, the analyzed embryos are not able to progress to transplantation.

On the other hand, a cantilever-based sensor for physical, chemical, and biological analysis has been reported. ${ }^{(12-14)}$ The mass of carbon, metal, and metal oxide nanoparticles was detected using silicon resonant cantilever-based mass sensors ${ }^{(15,16)}$ or silicon nanopillar resonators with sub-femtogram mass resolution. ${ }^{(17,18)}$ We have also developed a self-sensing high-sensitivity sensor using a piezoresistive cantilever, ${ }^{(19)}$ and have succeeded in antibody-antigen reaction measurement with the mass sensitivity of $190 \mathrm{fg} / \mathrm{Hz} .{ }^{(20)}$ Furthermore, we fabricated a holetype piezoresistive cantilever using a focused ion beam (FIB), and the high sensitivity of 23.1 $\mathrm{fg} / \mathrm{Hz}$ was obtained. ${ }^{(21)}$ In the cell or embryo, it is suggested that mass will change as a result of the transport of water, proteins, lipids, carbohydrates, and nucleic acids through the cell membrane. ${ }^{(22,23)}$ The total mass of single or multiple adherent mammalian cells under culture conditions was measured with an optically excited microresonator based on the cantilever sensor with picogram mass sensitivity. ${ }^{(24)}$ The mass change of the embryo, therefore, will be measured using a piezoresistive cantilever. In this study, we fabricated a holder-type piezoresistive cantilever using a FIB to place and fix an embryo on the cantilever, and we demonstrated the first measurement of a single mouse embryo mass.

\section{Mass Measurement with Cantilever Biosensor}

When we vibrate a microcantilever using an exciting piezoactuator that supports the cantilever base, the cantilever equation of motion is given by

$$
M \frac{d^{2} z}{d t^{2}}+\frac{M \omega_{0}}{Q} \frac{d z}{d t}+k z=F \sin \omega t
$$

where $M$ is the effective mass of the cantilever, $z$ is the deflection of the cantilever top, $\omega_{0}$ is the resonance frequency under the free-standing condition, $Q$ is the quality factor, $k$ is the spring constant, and $F \sin \omega t$ is the external vibration force from the exciting piezoactuator. ${ }^{(25)}$ The cantilever is vibrated at the resonance frequency, $\omega$, which is given by

$$
\omega=\sqrt{\frac{k\left(2 Q^{2}-1\right)}{M Q^{2}}} .
$$


This equation indicates that the resonance frequency changes as the cantilever mass changes, and that the frequency change depends on the spring constant and the $Q$ factor. When we vibrate the cantilever in a culture medium of high viscosity as the buffered solution, $Q$ becomes small compared with that in the low-viscosity environment of air. The change in resonance frequency, $\Delta \omega$, is given by the total differential of Eq. (2).

$$
\Delta \omega=\frac{\omega}{Q\left(2 Q^{2}-1\right)} \Delta Q+\frac{f}{2 k} \Delta k-\frac{\omega}{2 M} \Delta M
$$

$M$ is given by $M=n m$, where $n$ is a constant and $m$ is the mass of the cantilever. Rectangular cantilevers have been reported to have $n=0.24$. $^{(26)}$ The spring constant change, $\Delta k$, is assumed to be zero because the spring constant does not change. Therefore, $\Delta \omega$ is given by

$$
\Delta \omega=\frac{\omega}{Q\left(2 Q^{2}-1\right)} \Delta Q-\frac{\omega}{2 m} \Delta m
$$

Depending on the material fixed on the cantilever, the mass, $m$, changes to $m+\Delta m$, and the resonance frequency, $\omega$, changes to $\omega-\Delta \omega$. From Eq. (4), the mass change, $\Delta m$, is given by

$$
\Delta m=\frac{2 m}{Q\left(2 Q^{2}-1\right)} \Delta Q-\frac{2 m}{\omega} \Delta \omega
$$

In order to detect small $\Delta m$, it is necessary to use a cantilever with a small mass, a high resonance frequency, and a high $Q$ factor, and to detect small changes in the $Q$ factor and resonance frequency with a fine resolution. The microcantilever satisfies the requirements of small mass and high resonance frequency, and the femtogram mass measurement system also satisfactorily detects the small changes in the $Q$ factor and resonance frequency. However, if the mass of the target material is heavier than the cantilever mass, Eqs. (3) to (5) are not applicable to the estimation of the target mass, because the $Q$ factor and the $k$ change drastically. We also must consider the changes in the center of total mass (cantilever mass and target mass). In this research, as the mouse embryo was not fixed to the cantilever, only a small amount of the embryo mass was effectively weighted to the cantilever owing to effect of buoyancy, which we discuss later in this paper.

\section{Experimental Procedure}

\subsection{Design of holder-type piezoresistive cantilever using finite-element modeling (FEM)}

To hold and fix a single embryo on the piezoresistive cantilever, we proposed to fabricate a holder structure on the cantilever. Figure 1(a) shows a schematic design of a commercial piezoresistive cantilever (NPX1CTP004, SII Nanotechnology), which has a length of $120 \mu \mathrm{m}$, a width of $50 \mu \mathrm{m}$, a thickness of about $5 \mu \mathrm{m}$, a spring constant of $40 \mathrm{~N} / \mathrm{m}$, and a small rectangular 


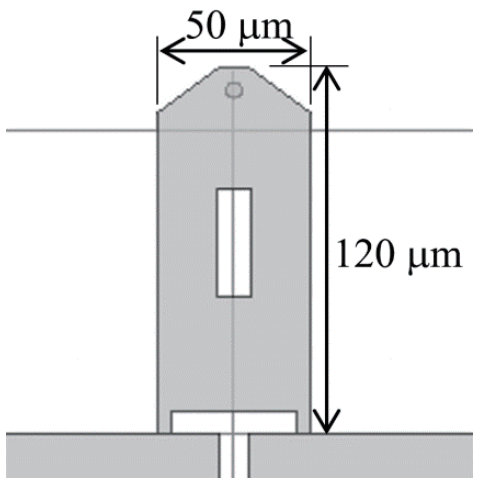

(a)

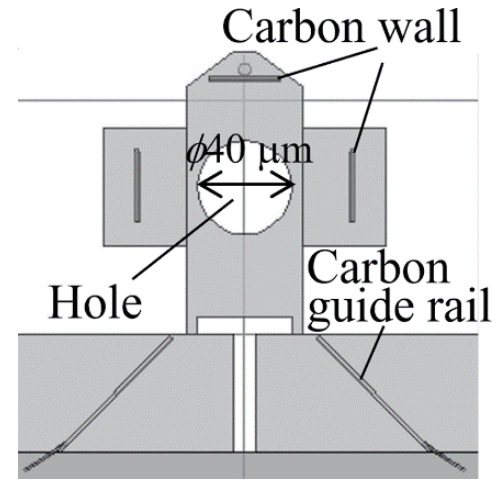

(b)

Fig. 1. Schematics of (a) commercial piezoresistive cantilever (NPX1CTP004, SII Nanotechnology) and (b) type E cantilever designs.

hole of about $10 \times 35 \mu \mathrm{m}^{2}$ at the center of the cantilever. A piezoresistor is embedded in the neck of the cantilever base, and the piezoresistance $R$ and sensitivity due to cantilever deflection, $\Delta R / R$, are $629 \Omega$ and $6.83 \times 10^{-5} \mathrm{~nm}^{-1}$, respectively. Figure 1(b) shows a schematic design of a holder-type piezoresistive cantilever of type E, which has two carbon wings, a hole $40 \mu \mathrm{m}$ in diameter, three carbon walls, and guide rails to prevent the embryo from falling off. Although prototypes of several kinds of holder-type cantilevers were made before the type $\mathrm{E}$ cantilever was fabricated, as shown in Fig. S1 (Appendix), we could only place the embryo on the type E cantilever. The details will be described later.

To confirm highly sensitive mass detection using the holder-type piezoresistive cantilever, the resonance frequency was calculated by computer simulation using the commercially available software COMSOL Multiphysics (COMSOL, Inc.). This software solves partial differential equations of physical parameters by FEM. In the simulation, the 3D structure of the cantilever is designed by the computer-aided design system, and the material properties, boundary conditions, and mesh size of the segment region are set in the designed 3D structures. Then, the software executes the calculations of the finite-element analysis together with adaptive meshing and error control using a variety of numerical solvers. It can perform original frequency analysis or stationary-state analysis.

\subsection{Fabrication of holder-type piezoresistive cantilever}

We fabricated a hole, carbon wings, plates, and guide rails for securing an embryo on a commercial piezoresistive cantilever (NPX1CTP004, SII Nanotechnology) by FIB (SMI500, SII Nanotechnology) processing with the accelerating voltage of $30 \mathrm{kV}$. The hole was fabricated by $\mathrm{Ga}$ ion beam etching, and the carbon structures were fabricated by ion-beam-assisted carbon deposition using a phenanthrene source. The structures to be fabricated were programed into the controlling PC of the FIB as bitmap images, and the fabrication position was adjusted on a scanning ion microscope (SIM) image. We selected the ion beam currents of $2 \mathrm{pA}, 400 \mathrm{pA}$, and $10 \mathrm{nA}$ for SIM observation, carbon deposition, and ion beam etching, respectively. The 
cantilever structure after FIB fabrication was confirmed by SIM observation with the tilt angle of $45^{\circ}$ and a top view using the sample stage tilting system.

\subsection{Embryo mass measurement system}

The prototype holder-type cantilever was mounted on a piezoactuator to vibrate the cantilever using a function generator, and the piezoresistor in the cantilever base was connected to a Wheatstone bridge and amplifier circuits, as shown in Fig. 2. The frequency properties of the cantilevers were measured by the frequency sweep method in the frequency range from 50 to $400 \mathrm{kHz}$ in $10 \mathrm{~Hz}$ steps and the range from 50 to $150 \mathrm{kHz}$ in $1 \mathrm{~Hz}$ steps using the piezoactuator and the bridge circuits. When we measured the embryo mass, the cantilever was submerged in the culture medium, then the embryo was placed on the holder position of the cantilever using a finely sharpened glass pipet. The embryo mass was obtained from the resonance frequency and change in the $Q$ factor after embryo placement, using Eq. (4).

\subsection{Preparation of mouse embryo obtained by IVF}

The mouse embryo obtained by IVF was prepared by the reported method with the following procedure. ${ }^{(3)}$ Male ICR mice (9-10 weeks old; Charles River JAPAN Inc.) were sacrificed by cervical dislocation. Sperm collected from caudal epididymides of ICR male mouse was incubated for $1-2 \mathrm{~h}$ at $37{ }^{\circ} \mathrm{C}$ and $5 \% \mathrm{CO}_{2}$. Female BDF1 mice (9-10 weeks old; Charles River JAPAN Inc.) were kept for $48 \mathrm{~h}$ after intraperitoneal injection of $5 \mathrm{IU}$ pregnant mare's serum gonadotropin (PMSG; ASKA Pharmaceutical, Japan) and subsequently sacrificed by cervical dislocation $12 \mathrm{~h}$ after intraperitoneal injection of $10 \mathrm{IU}$ human chorionic gonadotropin (hCG; ASKA Pharmaceutical, Japan) for ovarian hyperstimulation. Oviducts were excised, and ova were drawn with a needle. The ova were introduced into a drop of human tubal fluid (HTF) with sperm for IVF. About $5 \mathrm{~h}$ after insemination, treated ova were washed and moved from the fertilization medium to a drop of potassium simplex optimized medium (KSOM; Arc Resources, Japan) for culture. About $20 \mathrm{~h}$ after fertilization, a 2-cell-stage embryo was formed by the first cell division. A single 2-cell-stage mouse embryo was measured using the prototype

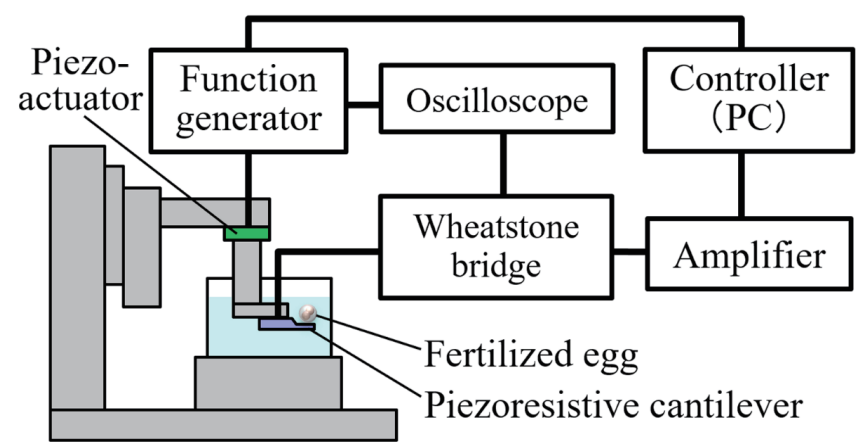

Fig. 2. (Color online) Schematic of embryo mass measurement system. 
cantilever in a culture medium of Dulbecco's phosphate-buffered saline [D-PBS (-), GIBCO] with $5 \%$ calf serum (CS, GIBCO).

\section{Results and Discussion}

The holder-type piezoresistive cantilevers were fabricated using commercial piezoresistive cantilevers, shown in the SIM image in Fig. 3(a). Figure 3(b) shows a SIM image of the holdertype cantilever fabricated by FIB processing in accordance with the designs in Fig. 1(b). The figure is tilted at an angle of $45^{\circ}$. We confirmed that the type E cantilever had carbon wings, a hole of $40 \mu \mathrm{m}$ diameter, three carbon walls, and guide rails on the cantilever.

The resonance frequency and $Q$ factor of the type E cantilever before and after FIB processing in air changed from 257 to $228 \mathrm{kHz}$ and from 257 to 227 , respectively, as shown in Fig. 4(a). These reductions in the resonance frequency $f_{\max }$ and the $Q$ factor were caused by the increase in mass owing to the addition of carbon wings and walls. The resonance frequency

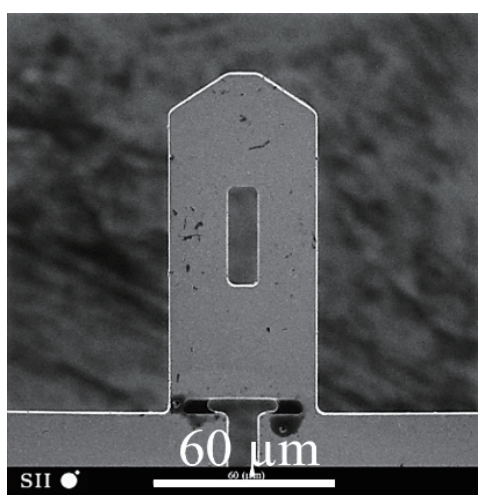

(a)

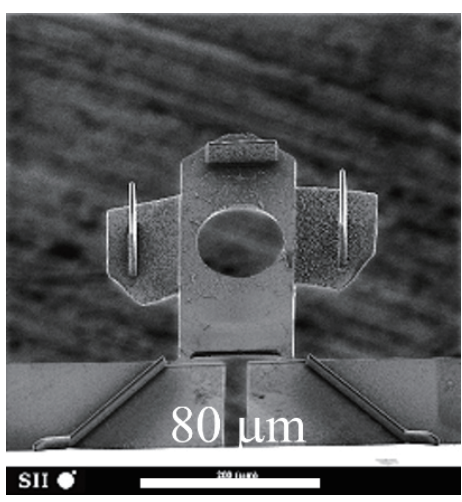

(b)

Fig. 3. SIM images of piezoresistive cantilever: (a) commercial one (NPX1CTP004, SII Nanotechnology) and (b) type E cantilever with image tilt angle of $45^{\circ}$.

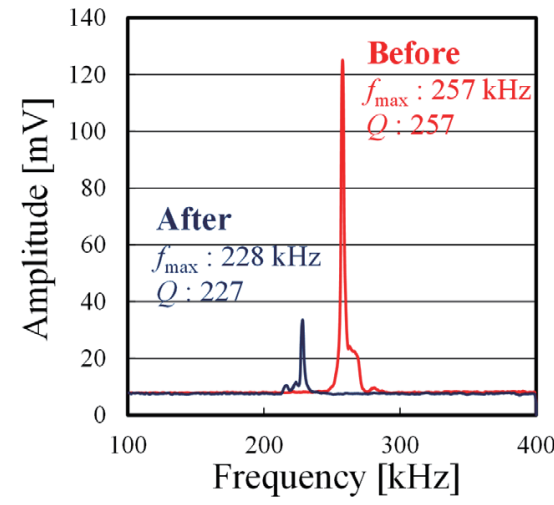

(a)

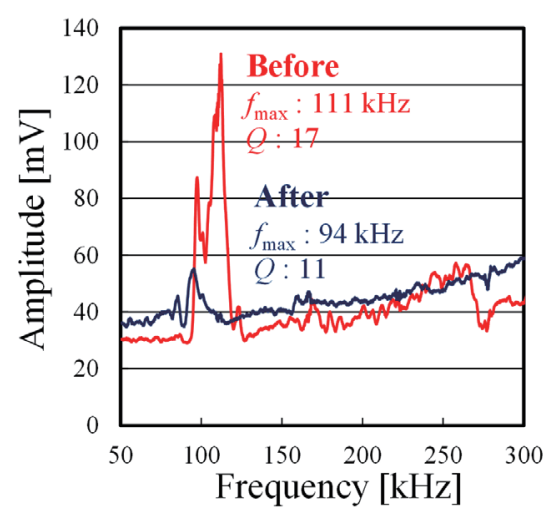

(b)

Fig. 4. (Color online) Frequency spectra of holder-type piezoresistive cantilever type E: (a) in air and (b) in water. 
and $Q$ factor before and after FIB processing in water changed from 111 to $94 \mathrm{kHz}$ and from 17 to 11, respectively, as shown in Fig. 4(b). These reductions in $f_{\max }$ and the $Q$ factor were also caused by the additional mass due to carbon deposition.

Figure 5(a) shows an optical microscope image of 2-cell-stage mouse embryos in culture medium. We can confirm that the embryo diameter is about $100 \mu \mathrm{m}$, and all embryos have 2 cells with the inner cell mass within the embryo membrane. Then, we drew up a single embryo using a finely sharpened glass pipet and placed it on the holder-type cantilever. Figure 5(b) shows an optical microscope image of the type E cantilever after the placement of a single 2-cell-stage mouse embryo in culture medium. First, the embryo was placed on the cantilever base, the bottom position in Fig. 5(b). Subsequently, it was moved to the holder positon by floating in medium using the glass pipet, and it is confirmed that the mouse embryo was placed at the holder position in the cantilever center between the carbon walls.

Figure 6(a) shows the frequency spectra of the cantilever before (solid curve) and after (thick solid curve) embryo placement. The resonance frequency and the $Q$ factor of the

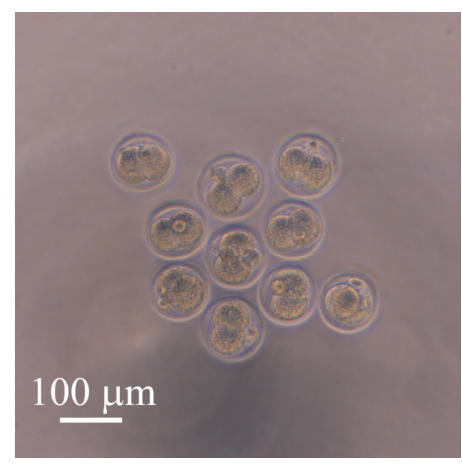

(a)

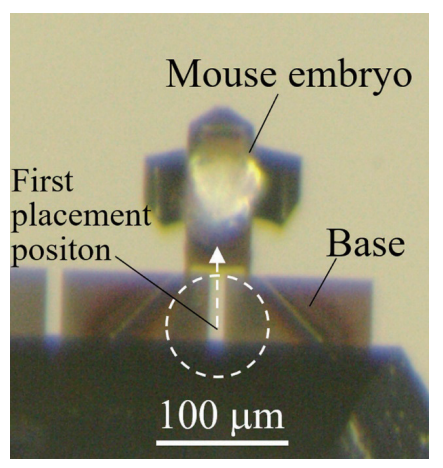

(b)

Fig. 5. (Color online) Optical microscope images of (a) 2-cell-stage mouse embryos in culture medium and (b) type E cantilever after placement of single 2-cell-stage mouse embryo in culture medium.

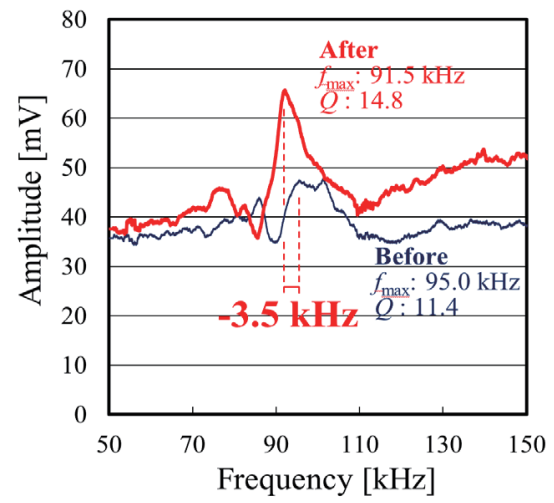

(a)

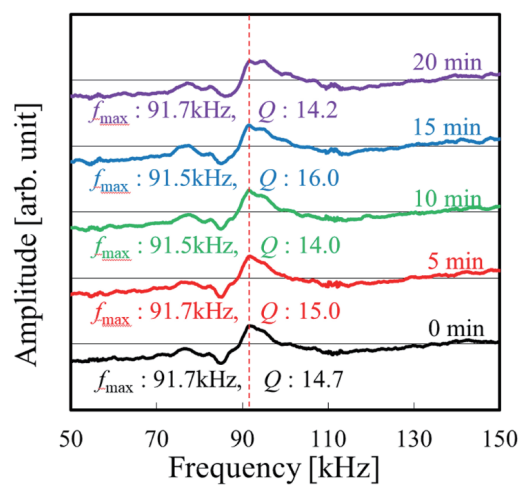

(b)

Fig. 6. (Color online) Frequency spectra of type E cantilever: (a) before and after single mouse embryo placement and (b) time from 0 to $20 \mathrm{~min}$ after mouse embryo placement. 
cantilever before and after the placement changed from 95.0 to $91.5 \mathrm{kHz}$ and from 11.4 to 14.8 , respectively. From the frequency change of $-3.5 \mathrm{kHz}$, the $Q$ factor change of 3.4 and $m$ of $57.4 \mathrm{ng}$, which were calculated using the volume and density of the type E cantilever, the mouse embryo mass was estimated to be about 4.4 ng using Eq. (5). Since the mouse embryo is submerged in the culture medium, the density of the mouse embryo must be higher than that of the culture medium. Thus, we can estimate the mass of mouse embryo to be greater than $520 \mathrm{ng}$. This value was calculated from the volume of a sphere with a diameter of $100 \mu \mathrm{m}$ and density of $1.004 \mathrm{~g} / \mathrm{cm}^{3}$, which was measured using a Gay-Lussac-type pycnometer at $26{ }^{\circ} \mathrm{C}$ for the culture medium of D-PBS with 5\% CS. Therefore, the estimated embryo mass in our experiment is over 100 times smaller than the calculated mass. Note that the embryo was not fixed to the cantilever, and the embryo mass was not effectively weighted to the cantilever. When the embryo was not fixed to the cantilever, buoyancy act on the embryo. The buoyancy was calculated from the volume of the embryo and the density of culture medium. Therefore, the estimated embryo mass of $4.4 \mathrm{ng}$ is an apparent mass obtained as the deferential between real mass and buoyancy. In order to explore this result, we calculated the vibration mode of the holder-type cantilever in water using FEM simulation of COMSOL Multiphysics with an acoustics module. The cantilever model was placed in a sphere of water and an absorbing boundary condition of the water surface was set. Figure 7(a) shows the simulation result of the type E cantilever at maximum deflection before embryo placement, where the physical parameters of the embryo were a diameter of $90 \mu \mathrm{m}$, density of $1027 \mathrm{~kg} / \mathrm{m}^{3}$, Poisson's ratio of 0.2 and Young's modulus of $33 \mathrm{kPa} .{ }^{(3)}$ The calculated resonance frequency of the cantilever is about $123 \mathrm{kHz}$. This value is larger than the measured value of $95.0 \mathrm{kHz}$, because the viscosity and density of the culture medium are slightly different from those of water, and the shape of the cantilever is slightly different from that of the designed model. Figure 7(b) shows a simulation results of the cantilever at maximum deflection after fixing the embryo at the contact edge of the circular hole. The calculated resonance frequency of the cantilever is about $2.3 \mathrm{kHz}$, which is much smaller than the measured value of $91.5 \mathrm{kHz}$ after embryo placement, because the large mass of the embryo was fixed to the cantilever. Thus, we calculated the vibration mode of the cantilever after embryo placement without fixing, as shown in Fig. 7(c). As the result, the embryo was found to be slightly moved from the circular hole to the base of the cantilever, and the embryo surface was distorted. The calculated resonance frequency of the cantilever is about $102 \mathrm{kHz}$. This value is smaller than the simulation result of $123 \mathrm{kHz}$ before embryo placement, and is larger than the $2.3 \mathrm{kHz}$ after fixing the embryo. Although the resonance frequency change of $-21 \mathrm{kHz}$ before and after embryo placement is larger than the experimental result of $-3.5 \mathrm{kHz}$, the frequency change will become close to the experimental value upon optimizing the physical parameters of the embryo and culture medium. As the embryo mass in the simulation is about $392 \mathrm{ng}$, the estimated embryo mass of $4.4 \mathrm{ng}$ is also 100 times smaller than the calculated mass in the simulation. In order to measure the embryo mass with highaccuracy, we will fabricate a large holder-type cantilever with a wide hole of nearly $100 \mu \mathrm{m}$ in diameter for fixing the embryo in future work. Furthermore, when we estimate the mass of the mouse embryo, we will simultaneously use the FEM computer simulation. 


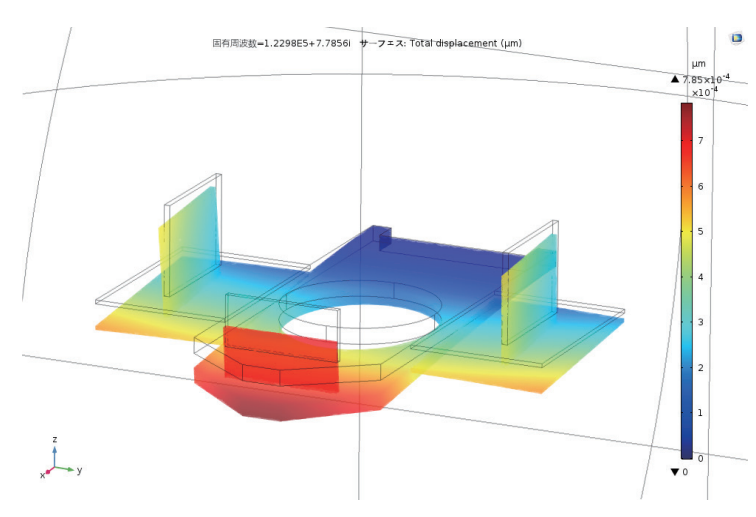

(a)

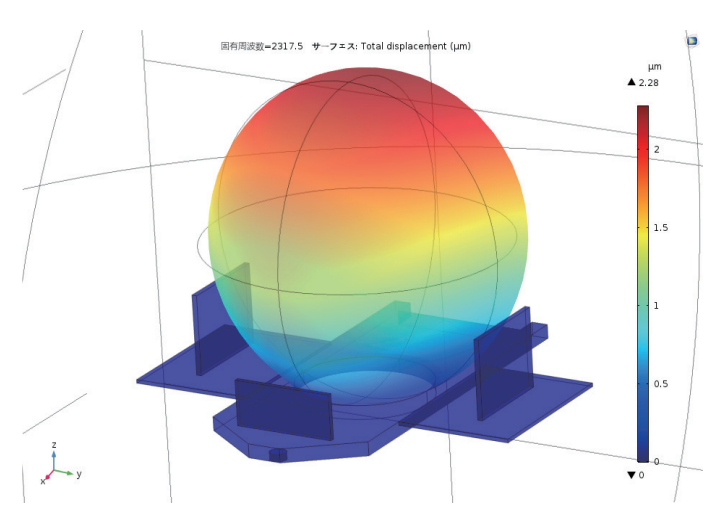

(b)

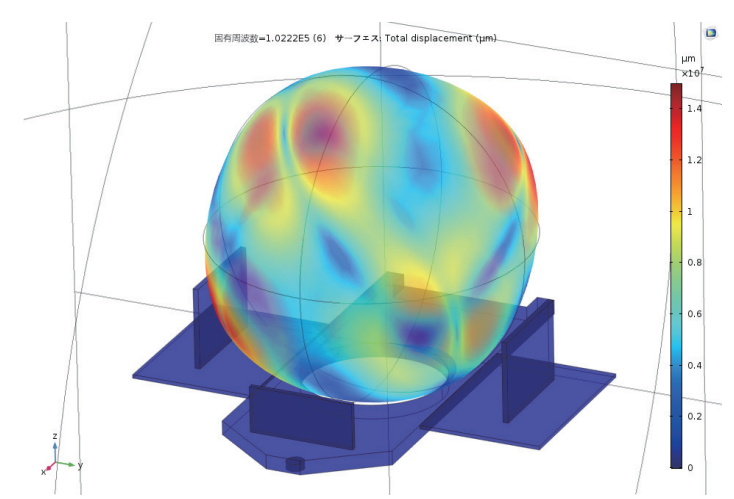

(c)

Fig. 7. (Color online) Three-dimensional images of simulation results for type E cantilever in water: (a) before embryo placement, (b) after fixing embryo to the hole edge of the cantilever, and (c) after embryo placement without fixing.

Figure 6(b) shows the frequency spectra of the holder-type cantilever obtained from 0 to $20 \mathrm{~min}$ in $5 \mathrm{~min}$ steps after mouse embryo placement. Although the shape of the spectra changed slightly between 10 and $15 \mathrm{~min}$, the resonance frequency was almost a constant value of $91.7 \mathrm{kHz}$ up to $20 \mathrm{~min}$. This result shows that the measurement time of $20 \mathrm{~min}$ is too short to monitor the embryo growth. To evaluate the holder-type cantilever system performance for embryo mass monitoring, we must modify or fabricate a holder-type cantilever with a suitable embryo fixing structure; then, we can try to monitor the embryo mass for 3-4 d.

\section{Conclusions}

A prototype of a novel embryo evaluation system was made by FIB fabrication of a holdertype piezoresistive cantilever and a monitoring system of the cantilever resonance frequency. Five holder-type cantilevers were fabricated and the frequency properties were evaluated by the frequency sweep method and FEM simulation. The type E cantilever was found to have a high mass sensitivity because it has a high resonance frequency and $Q$ factor in water, 94 $\mathrm{kHz}$ and 11, respectively. A single 2-cell-stage mouse embryo mass was measured using the 
type E cantilever in a culture medium of PBS with calf serum. The changes in the resonance frequency and $Q$ factor of the cantilever before and after embryo placement were $-3.5 \mathrm{kHz}$ and 3.4 , respectively. Then, the mouse embryo mass was estimated to be about $4.4 \mathrm{ng}$. Although this value is over 100 times smaller than the mass calculated from the volume of the mouse embryo and the density of the culture medium, it indicates the possibility of measuring the mass of a single mouse embryo using the holder-type cantilever sensor system.

\section{Acknowledgments}

This work was supported by JSPS KAKENHI Grant Number JP26282143.

\section{References}

1 L. L. Veeck: Atlas of the Human Oocyte and Early Conceptus, Vol. 2 (Williams \& Wilkins, Baltimore, 1991) p. 151.

2 D. K. Gardner, M. Lane, J. Stevens, T. Schlenker, and W. B. Schoolcraft: Fertil. Steril. 73 (2000) 1155.

3 Y. Murayama, J. Mizuno, H. Kamakura, Y. Fueta, H. Nakamura, K. Akaishi, K. Anzai, A. Watanabe, H. Inui, and S. Omata: Human Cell 19 (2006) 119.

4 Y. Murayama, M. Yoshida, J. Mizuno, H. Nakamura, S. Inoue, Y. Watanabe, K. Akaishi, H. Inui, C. E. Constantinou, and S. Omata: J. Mamm. Ova Res. 25 (2008) 8.

5 T. Sakata, I. Makino, and S. Kita: Eur. Biophys. J. 40 (2011) 699.

6 T. Sakata, A. Saito, J. Mizuno, H. Sugimoto, K. Noguchi, E. Kikuchi, and H. Inui: Anal. Chem. 85 (2013) 6633.

7 Y. Chen, G. Fan, H. Wu, Y. Wu, and A. Mitchell: J. Pharm. Biomed. Anal. 43 (2007) 99.

8 C. R. Ferreira, S. A. Saraiva, R. R. Catharino, J. S. Garcia, F. C. Gozzo, G. B. Sanvido, F. A. L. Santos, E. G. Lo Turco, J. H. F. Pontes, A. C. Basso, R. P. Bertolla, R. Sartori, M. M. Guardieiro, F. Perecin, F. V. Meirelles, J. R. Sangalli, and M. N. Eberlin: J. Lipid Res. 51 (2010) 1218.

9 R. F. Gonçalves, C. R. Ferreira, C. M. B. Orlandi, V. C. Sartori, H. N. Ferreira, F. C. Gozzo, S. A. Saraiva, E. J. Pilau, and M. N. Eberlin: Reprod. Fertil. Dev. 23 (2011) 160.

10 A. Tata, M. J. Sudano, V. G. Santos, F. D. C. Landim-Alvarenga, C. R. Ferreira, and M. N. Eberlina: J. Mass Spectrom. 48 (2013) 844.

11 C. R. Ferreira, L. S. Eberlin, J. E. Hallett, and R. G. Cooks: J. Mass Spec. 47 (2012) 29.

12 R. Berger, Ch. Gerber, H. P. Lang, and J. K. Gimzewski: Microelectron. Eng. 35 (1997) 373.

13 H. P. Lang, M. K. Baller, R. Berger, Ch. Gerber, J. K. Gimzewski, F. M. Battiston, P. Fornaro, J. P. Ramseyer, E. Meyer, and H. J. Güntherodt: Anal. Chim. Acta 393 (1999) 59.

14 J. Fritz, M. K. Baller, H. P. Lang, H. Rothuizen, P. Vettiger, E. Meyer, H. J. Guntherodt, Ch. Gerber, and J. K. Gimzewski: Science 288 (2000) 316-318.

15 H. S. Wasisto, S. Merzsch, A. Waag, I. Kirsch, E. Uhde, T. Salthammer, and E. Peiner: Microsyst. Technol. 18 (2012) 905.

16 H. S. Wasisto, S. Merzsch, A. Waag, E. Uhde, T. Salthammer, and E. Peiner: Sens. Actuators, A 202 (2013) 90.

17 Y. Lu, S. Peng, D. Luo, and A. Lal: Nature Com. 2 (2011) 578.

18 H. S. Wasisto, K. Huang, S. Merzsch, A. Stranz, A. Waag, and E. Peiner: Microsyst. Technol. 20 (2014) 571.

19 H. Sone, H. Okano, and S. Hosaka: Jpn. J. Appl. Phys. 43 (2004) 4663.

20 H. Sone, A. Ikeuchi, T. Izumi, H. Okano, and S. Hosaka: Jpn. J. Appl. Phys. 45 (2006) 2301.

21 A. H. Truong, H. Sone, T. Kawakami, and S. Hosaka: Key Eng. Mater. 534 (2013) 251.

22 D. Häussinger: Biochem. J. 313 (1996) 697.

23 F. Lang, G. L. Busch, M. Ritter, H. Völkl, S. Waldegger, E. Gulbins, and D. Häussinger: Physiol. Rev. 78 (1998) 247.

24 D. Martínez-Martín, G. Fläschner, B. Gaub, S. Martin, R. Newton, C. Beerli, J. Mercer, C. Gerber, and D. J. Müller: Nature 550 (2017) 500.

25 A. Kühle, A. H. Sørensen, and J. Bohr: J. Appl. Phys. 81 (1997) 6562.

26 G. Y. Chen, R. J. Warmack, T. Thundat, D. P. Allison, and A. Huang: Rev. Sci. Instrum. 65 (1994) 2532. 


\section{Appendix}

Prototypes of several kinds of holder-type cantilevers were made before type E cantilever fabrication. Figures S1(a)-S1(d) show the schematic designs (left) and SIM images (right) of type A to type D holder-type piezoresistive cantilevers. Type A has a square hole of $40 \times 40$ $\mu \mathrm{m}^{2}$ on the cantilever. Type B has two carbon wings on both sides of the cantilever. Type $\mathrm{C}$ has two carbon wings, a hole of $40 \mu \mathrm{m}$ diameter, and a carbon ring of $90 \mu \mathrm{m}$ diameter to prevent the embryo from falling off the cantilever. Type D has two carbon wings, a hole of 40 $\mu \mathrm{m}$ diameter, and four carbon walls to hold the embryo. We confirmed, by SIM observation, that those prototype holder-type cantilevers have structures similar to the designed shape.

The resonance frequency and $Q$ factor of the type A cantilever before and after FIB processing in air changed from 295 to $387 \mathrm{kHz}$ and from 171 to 221, respectively, as shown in Fig. S2(a). The increases in the resonance frequency and $Q$ factor were the result of reduced mass due to hole fabrication. The resonance frequency and $Q$ factor before and after FIB processing in water changed from 170 to $262 \mathrm{kHz}$ and from 16 to 12 , respectively, as shown in Fig. S2(b). Although the $Q$ factor was slightly decreased, the resonance frequency was increased because of the reduced mass due to hole fabrication. Therefore, the type A cantilever has the advantage of high sensitivity. However, we could not place a mouse embryo on the cantilever because the size of the square hole was too small compared with the embryo diameter.

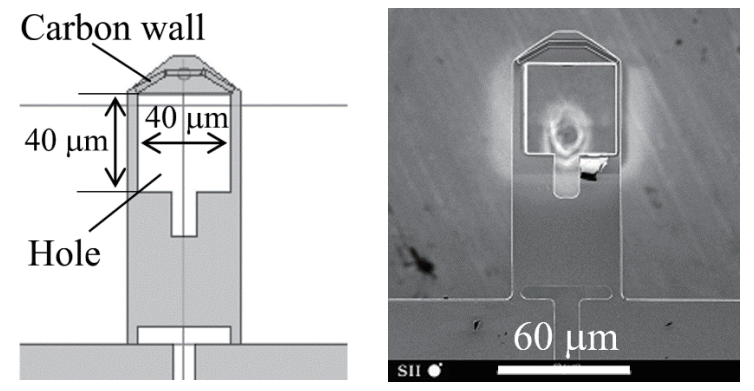

(a)

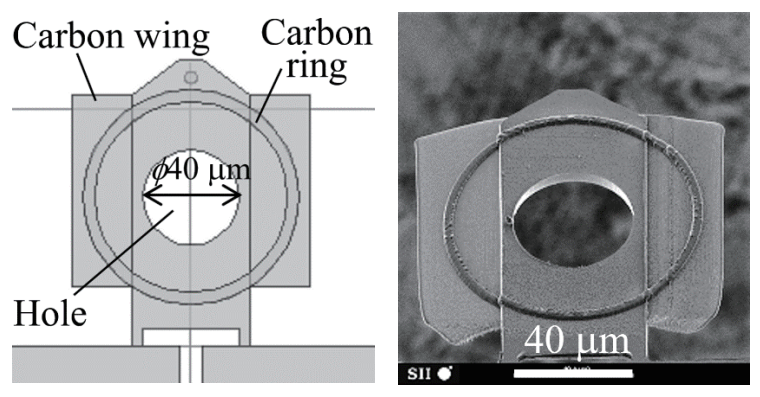

(c)
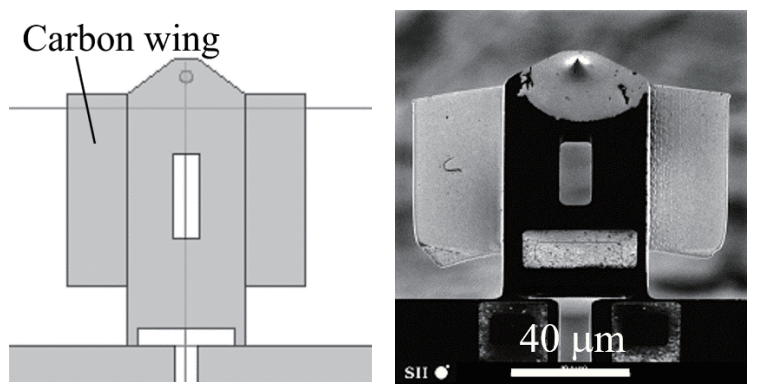

(b)
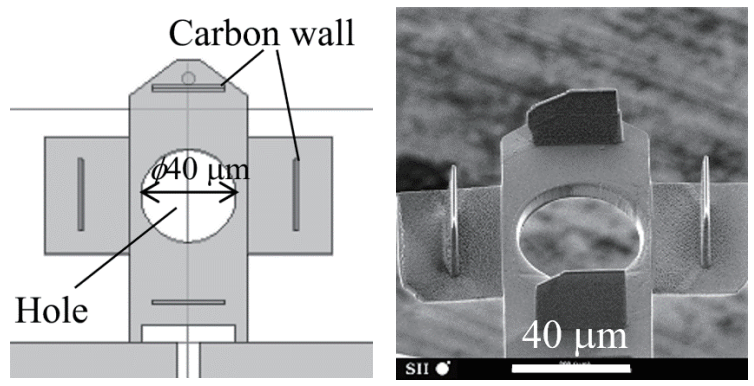

(d)

Fig. S1. Schematic of designs (left) and SIM images (right) of (a) type A, (b) type B, (c) type C, and (d) type D cantilevers. Images (b) to (d) have a tilt angle of $45^{\circ}$. 


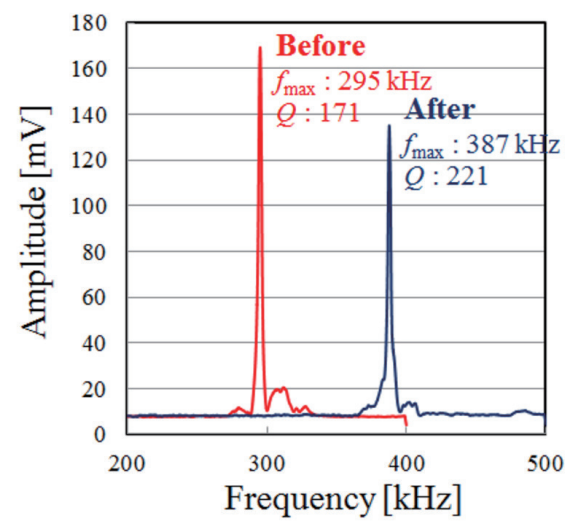

(a)

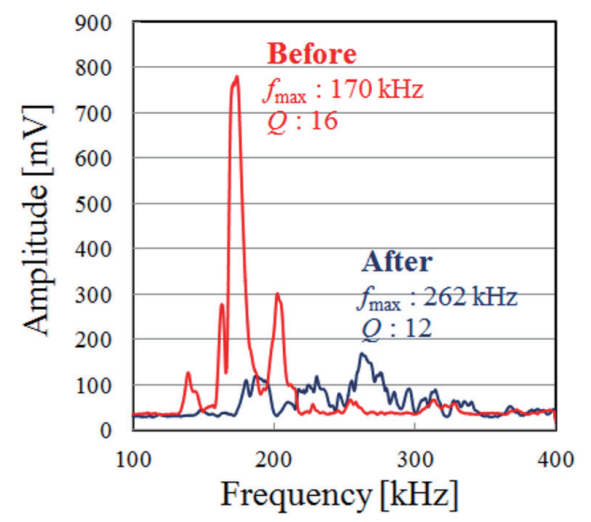

(b)

Fig. S2. (Color online) Frequency spectra of holder-type piezoresistive cantilever type A (a) in air and (b) in water.

Figure S3 shows the rates of change of the resonance frequency and $Q$ factor before and after FIB fabrication for type A to type E cantilevers in air (a) and in water (b). The resonance frequency and $Q$ factor of type A increased by about $30 \%$ in air owing to the reduced mass due to hole fabrication, as explained previously. On the other hand, those of type B to type E decreased in air owing to the additional mass of the carbon wings and walls. These trends were enhanced in water, as shown in Fig. S3(b). According to Eq. (5), a holder-type cantilever with higher resonance frequency and higher $Q$ factor is advantageous for obtaining a higher mass sensitivity. Figure $\mathrm{S} 3$ indicates that type $\mathrm{E}$ has a high mass sensitivity because it has a relatively high resonance frequency and $Q$ factor. The reason for the higher resonance frequency may be that the vertical carbon walls on the cantilever surface act to reduce the amount of turbulent fluid and to promote the stable vibration of the cantilever. Although type D also has the vertical carbon walls, the resonance frequency is lower than that of the type E cantilever. It seems that type D has an external vertical wall on the surface, and therefore, the resonance frequency is decreased because of the increased mass.

As we described above, although we attempted single mouse embryo placement on cantilevers of type A to type E, we were successful only on type E. The embryo fell off the cantilever owing to a lack of side protection walls on type A to type C. On the other hand, the external vertical wall prevented embryo transfer from the cantilever base to the holder position on type D. Thus, we used the type E cantilever to measure the mass of a mouse embryo.

To confirm that the prototype holder-type cantilevers have structures designed for high sensitivity, the resonance frequency was calculated by FEM simulation. Figure S4 shows the rates of change of the resonance frequency before and after FIB fabrication for type A to type $\mathrm{E}$ in air along with the experimental results for comparison. The rates of change of the resonance frequency before and after FIB fabrication in the experimental results are consistent with those of the simulation results for all types of cantilevers. This finding indicates that the holder-type cantilevers were precisely fabricated with the designed structure and operated with the required properties. The resonance frequencies of the type B to type E holder-type cantilevers were slightly decreased, because the masses of those cantilevers were increased as a result of the 

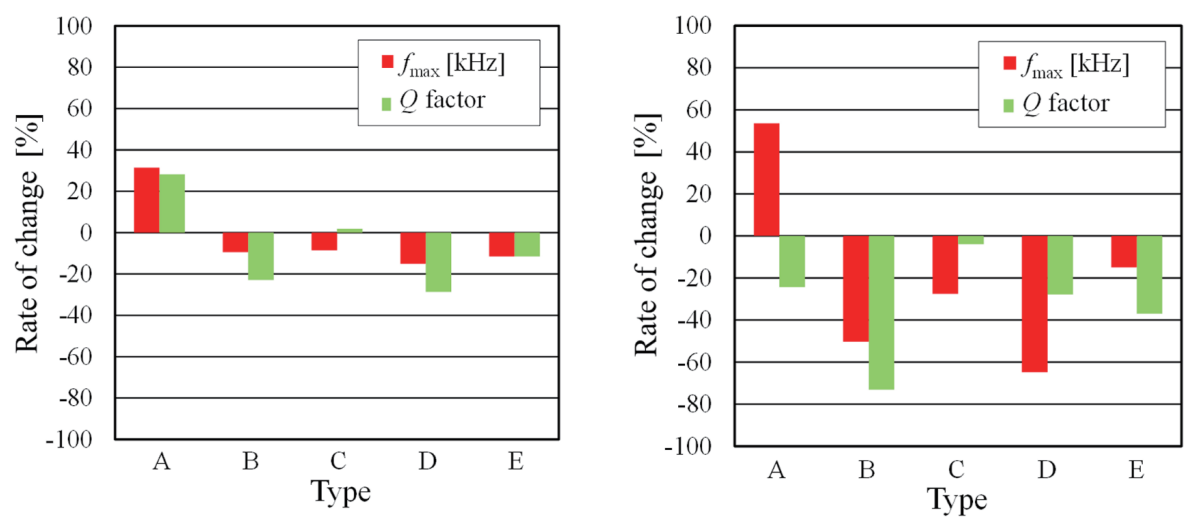

Fig. S3. (Color online) Rate of change of frequency properties before and after FIB processing (a) in air and (b) in water.

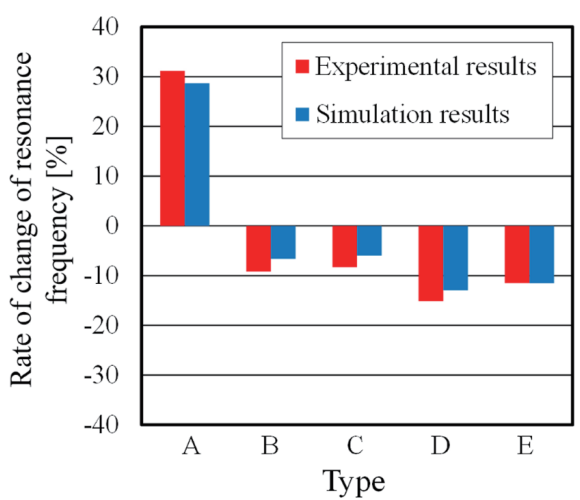

Fig. S4. (Color online) Rate of change of resonance frequency before and after FIB processing determined experimentally and by FEM simulation.

deposition of the carbon walls. However, as the decrease rate is about $10 \%$, the cantilever mass has a nominal influence on the measurement of embryo mass.

\section{About the Authors}

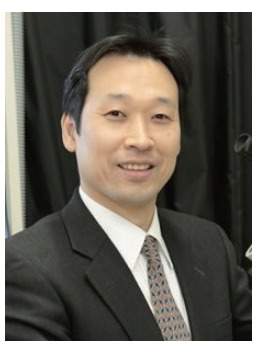

Hayato Sone received his B.S., M.S., and Ph.D. degrees from Tokyo University of Science, Japan, in 1993, 1995, and 2001, respectively. From 1999 to 2002, he was a special postdoctoral researcher at RIKEN, Japan. From 2002 to 2008, he was an assistant professor and, from 2008 to 2015, an associate professor at Gunma University, Japan. Since 2015, he has been a professor at Gunma University. His research interests are nanoscale measurement and fabrication, electronic devices, and cantilever-based and nanowire-based biosensors. (hayatosone@gunma-u.ac.jp) 


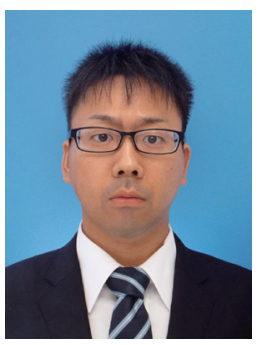

Tomoyuki Kawakami received his B.S. and M.S. degrees from Gunma University, Japan, in 2012 and 2014, respectively. Since 2014, he has been at Nissin Industry Co., Ltd., Japan. (tomoyuki-kawakami@nisshinkogyo.co.jp)

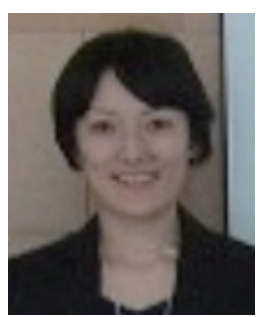

Akiko Saito has been a technical staff of The University of Tokyo since 2011. She has also been a bachelor course student of Tokyo University of Science since 2013. Her research interests are cellular analysis and biosensors. (saito@biofet.t.u-tokyo.ac.jp)

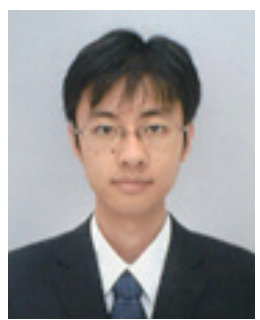

Yuki Sayama received his B.S. degree from Gunma University, Japan, in 2017. Since 2017, he has been a masters course student of Gunma University. His research interest is nanoscale cantilever-based biosensors.

(t171d046@gunma-u.ac.jp)

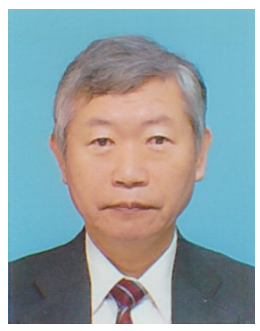

Sumio Hosaka received his B.S. degree from Yamanashi University, Japan, in 1971 and his Ph.D. degree from Waseda University, Japan, in 1983. He joined Hitachi Central Research Laboratory in 1971 and has been a professor at Gunma University since 2001 and a professor emeritus there since 2014. His research interests are MEMS, nanofabrication, nanodevices, and probe microscopy. (hosaka@gunma-u.ac.jp)

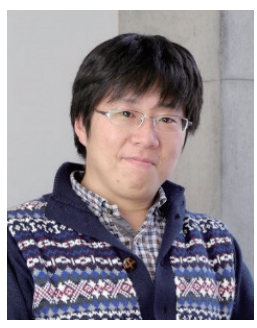

Toshiya Sakata received his B.S., M.S., and Ph.D. degrees from Osaka University, Japan, in 1998, 2000, and 2003, respectively. From 2003 to 2006, he was a researcher at National Institute for Materials Science (NIMS), Japan. From 2006 to 2011, he was a lecturer at the University of Tokyo, Japan. Since 2011, he has been an associate professor at the University of Tokyo. His research interests are biosensing materials and biosensors.

(sakata@biofet.t.u-tokyo.ac.jp) 